\title{
Highly hydrophilic water-insoluble nanofiber composite as an efficient and easily-handleable adsorbent for a rapid adsorption of cesium from radioactive wastewater
}

\author{
Hyunsik Bang, ${ }^{\text {a }}$ Kei Watanabe, ${ }^{a}$ Ryu Nakashima, ${ }^{\text {a Wei Kai }},{ }^{b}$ Kyung-Hun Song, ${ }^{c}$ Jung Soon Lee, ${ }^{\mathrm{d}}$ Mayakrishnan
} ${ }_{5}$ Gopiraman, ${ }^{a}$ and Ick-Soo Kim*a

\author{
Received (in $X X X, X X X)$ Xth $X X X X X X X X X 20 X X$, Accepted Xth $X X X X X X X X X 20 X X$ \\ DOI: 10.1039/b000000x
}

Herein, we report a new Prussian blue nanoparticle (PBNPs) incorporated polyvinyl alcohol (PVA)

10 composite nanofiber ( $c$-PBNPs/PVA) for a rapid adsorption of cesium (Cs) from the radioactive

wastewater. Initially, various electrospinning parameters such as solvent, PVA wt\%, PBNPs wt\% and

glutaraldehyde (GA) wt \% were extensively optimized to obtain a better physicochemical property of the $c$-PBNPs/PVA. In order to improve the water insoluble nature of the PVA, a post cross-linking was carried out for the $c$-PBNPs/PVA using glutaraldehyde (GA) and $\mathrm{HCl}$ vapor as cross-linker and catalyst,

15 respectively. SEM images revealed smooth and continuous morphology of the $c$-PBNPs/PVA composite nanofibers with diameters of 200-300 nm and lengths up to several millimeters. TEM images confirmed homogeneous dispersion and well incorporation of PBNPs into PVA matrix. The amorphous nature of the $c$-PBNPs/PVA was confirmed by the XRD analysis. FT-IR spectra showed successful cross-linking of PVA with GA. It was found that the prepared composite nanofiber is highly hydrophilic and water-

20 insoluble. The $c$-PBNPs/PVA showed an excellent and faster Cs adsorption rate of $96 \%$ after only 100 min. These results are well comparable to those previous reported. After the Cs adsorption test, the $c$ PBNPs/PVA composite nanofiber can be easily separated from the wastewater.

\section{Introduction}

A catastrophic earthquake and tsunami occurred on March 11, ${ }_{25} 2011$, which caused a huge destruction in northeastern Japan. Undesirably, the Fukushima Daiichi Nuclear Power Plant (FDNPP) was also severely damaged. ${ }^{1}$ As a consequence, there have been continued spills of radioactive isotopes such as ${ }^{90} \mathrm{Sr}$, ${ }^{131} \mathrm{I},{ }^{134} \mathrm{Cs}$ and ${ }^{137} \mathrm{Cs}$ contaminated water from the FDNPP. ${ }^{2}$

30 Among the radioactive materials, radiocesium has a long-term of problem due to its long physical half-life (2 years for ${ }^{134} \mathrm{Cs}$ and 30.1 years for ${ }^{137} \mathrm{Cs}$ ) and its high biological availability. ${ }^{3,4}$ Indeed, this radiocesium $\left({ }^{134} \mathrm{Cs}\right.$ and $\left.{ }^{137} \mathrm{Cs}\right)$ is highly harmful to the living organisums. To overcome this issue, several Cs adsorbents have 35 been reported but only very few of them are highly efficient and selective. Among them, ferric hexacyanoferrate $\left(\mathrm{Fe}_{4}{ }^{\mathrm{III}}\left[\mathrm{Fe}^{\mathrm{II}}(\mathrm{CN})_{6}\right]_{3}\right)$ is also known as Prussian blue (PB), often used as an efficient adsorbent for the removal of radioactive $\mathrm{Cs}$ from the wastewater. ${ }^{5,6}$ Very recently, the Japanese National 40 Institute of Advanced Industrial Science and Technology (AIST) has developed PB nanoparticles (PBNPs) with the particle size of 10-20 nm. ${ }^{7}$ Interestingly, due to the high specific surface area, the PBNPs has showed an excellent adsorption efficiency of Cs (about 100\%). ${ }^{8}$ However, since the size of the PBNPs is too small, 45 the recovery of the PBNPs (after the adsorption of Cs) is very complicated which causes more problems to the living organisms and the environment. ${ }^{9}$ Reda et al., ${ }^{10}$ has prepared magnetic hexacyanoferrate(II) polymeric nanocomposite for the separation of Cs from radioactive wastewater. However, during the process, 50 these materials often suffer from the slower Cs adsorption rate and leaching of PBNPs from the polymeric supports into wastewater. Very recently, carbon materials have been played a tremendous role as support for various MNPs including PBNPs. ${ }^{11,12}$ Hongjun et al., ${ }^{13}$ has prepared magnetic Prussian 55 blue/graphene oxide nanocomposites for removal of radioactive Cs from wastewater. In spite of the higher activity, these composite materials are highly expensive and toxic. More importantly, recovery of these nanocmposites is complicated and expensive. ${ }^{14}$ Therefore, developing an efficient, stable and easily 60 handlable PBNPs-based material for the removal of Cs remains an highly demading issue.

Recently, polymer nanofibers prepared via electrospinning technique have played tremendous role as a support in a wide range of applications. ${ }^{15}$ Particularly, the metal NPs-based 65 composite nanofibers have been attracting more attention in various fields because of their high surface area, costeffectiveness and easy-handling. ${ }^{16}$ Certainly, metal NPs-based PVA composite nanofibers have been extensively used for several applications. ${ }^{17,18}$ In fact, PVA is largely available, highly 70 hydrophilic, easily processable, biocompatible, non-toxic and 
chemical resistive. Dian and co-workers ${ }^{19}$ have prepared AgNPs embedded electrospun PVA nanofibers composite (AgNPs/PVA) for the SERS study applications. They found that the AgNPs/PVA composite is highly responsible and the composite 5 can be easy handled and disposed. Based on the previous reports, we presumed that the PBNPs based on PVA nanocomposite can exhibit higher activity and overcome the drawbacks such as slower Cs adsorption rate, less stabllity and the defficulties of handling. Herein, we report a new PBNPs-based PVA composite 10 nanofiber ( $c$-PBNPs/PVA) for the adsorption of $\mathrm{Cs}$ from radioactive wastewater. The resultant nanocomposite (cPBNPs/PVA) was completely characterized by various micro and spectroscopic methods. Inductive coupled plasma-mass spectroscopy (ICP-MS) was used to measure the Cs adsorption 15 activity of the $c$-PBNPs/PVA in wastewater.

\section{Experimental}

\section{Materials}

PVA [degree of hydrolysis $=88 \%$, degree of polymerization (DP) $\left.2_{20}=1700\right]$ was provided by Kuraray Co. Ltd., Japan. Aqueous solution containing $8 \mathrm{wt} \%$ of PBNPs (particle size: 10-20 nm) was provided by Kanto Chemical Co., Inc, Japan. Glutaraldehyde solution (GA, 50\% in water) was purchased from Sigma-Aldrich. $37 \% \mathrm{HCl}, 99.9 \% \mathrm{CsNO}_{3}$ and $2 \% \mathrm{HNO}_{3}$ were purchased from

${ }_{25}$ Wako pure chemicals, Japan. All chemicals were used asreceived without further purification.

\section{Preparation of $c$-PBNPs/PVA composite nanofiber}

\section{Preparation of $P V A / P B N P S / G A$ solution}

30 In order to prepare a good PBNPs-based PVA composite nanofiber, dispersibility of the PBNPs into the PVA solution is most important. First, a $1.2 \mathrm{~g}$ of PVA (12 wt\%) was dissolved in $10 \mathrm{~mL}$ of distilled water and the solution mixture was stirred at $80^{\circ} \mathrm{C}$ for $3 \mathrm{~h}$. After cooling to $27^{\circ} \mathrm{C}$, a $24 \mathrm{wt} \%$ of PBNPs (based 35 on the wt $\%$ of PVA solution) was mixed into the resultant PVA solution $(12 \mathrm{wt} \%)$ and stirred at $27^{\circ} \mathrm{C}$ for $24 \mathrm{~h}$. Finally, a 12.5 wt $\%$ of GA (based on the wt $\%$ of PVA) was mixed into the above resultant mixture (PVA/PBNPs solution) to obtain a homogeneous PVA/PBNPs/GA solution. The resultant 40 PVA/PBNPs/GA solution was used as an electrospinning solution for the preparation of nanofiber composite.

\section{Electrospinning}

A high-voltage power supply (Har-100*12, Matsusada Co., ${ }_{45}$ Tokyo, Japan), capable of generating voltages up to $100 \mathrm{kV}$, was used as the source of the electric field. The above obtained PVA/PBNPs/GA solutions were poured in a $5 \mathrm{~mL}$ plastic syringe attached to a capillary tip of about $0.6 \mathrm{~mm}$ in inner diameter. The $\mathrm{Cu}$ wire connected to an anode was inserted into the polymer 50 solution, and a cathode was attached to a grounded rotating metallic collector wrapped with cellulose nonwoven. The tip to collector distance (TCD) was $12 \mathrm{~cm}$ and the applied voltage was $10 \mathrm{kV}$. All the processes were carried out at $27^{\circ} \mathrm{C}$.

\section{Cross-linking process}

${ }_{55}$ Since the PVA is highly soluble in water, a post cross-linking was carried out for the resultant nanofiber composites (PBNPs/PVA/GA nanofiber) to improve the water resistance property of the PVA. In the typical experiment, all the obtained electrospun nanofibers were exposed to $\mathrm{HCl}$ vapor for $30 \mathrm{~s}$. In 60 order to produce $\mathrm{HCl}$ vapor, Conc. $\mathrm{HCl}$ was heated at $30^{\circ} \mathrm{C}$. Scheme 1 shows the schematic illustration for the preparation of $c$-PBNPs/PVA composite nanofibers.

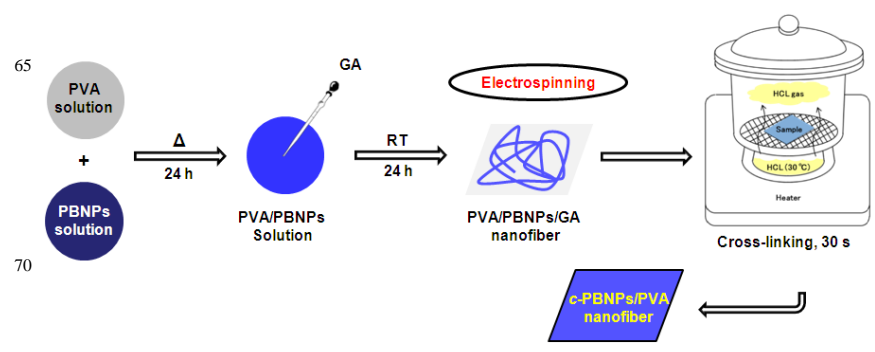

Scheme 1 Schematic illustration for the preparation of $c$ PBNPs/PVA composite nanofiber.

75

\section{Characterization}

PVA solution properties such as viscosity, surface tension and electrical conductively were measured with a viscometer (DV-1, Brookfield Co., Stoughton, MA, USA), a surface tension meter ${ }_{80}$ (CBVP-Z, Kyowa Interface Science Co., Ltd, Japan) and a conductivity meter (D-54, HORIBA, Ltd., Japan), respectively. The morphology of electrospun composite nanofibers was characterized by scanning electron microscopy (SEM, JSM6010LA, JEOL, Japan) and transmission electron microscopy 85 (TEM, 2010 Fas TEM, JEOL, Japan, accelerating voltage 200 $\mathrm{kV})$. The crystalline nature of the electrospun composite nanofibers was investigated using wide angle X-ray diffraction (XRD, Rotaflex RTP300, Rigaku, Japan) operating the diffractometer at $40 \mathrm{kV}$ and $150 \mathrm{~mA}$, using $\mathrm{Cu}$ Ka radiation. The 90 scans were recorded over the range $2 \theta=10-60^{\circ}$. To confirm the cross-linking of PVA with GA, Fourier Transform Infrared spectra (FT-IR, IRPrestige-21, Shimadzu Co., Japan) were recorded for composite nanofibers. The FT-IR spectra were recorded from 600 to $4000 \mathrm{~cm}^{-1}$ at a resolution of $4 \mathrm{~cm}^{-1}$. The $\mathrm{Cs}$ 95 adsorption activity of $c$-PBNPs/PVA was measured by using Inductively Coupled Plasma Mass Spectrometry (ICP-MS, NexION 300D, PerkinElmer Japan Co., Ltd., Japan). The leaching of PBNPs from the $c$-PBNPs/PVA was analyzed by UVVis spectro-photometer (Hitachi U-3500 UV-Vis 100 spectrophotometer).

\section{Cs adsorption test}

A $1.0 \mathrm{ppm}$ of Cs aqueous solution was prepared by adding $1.5 \mathrm{mg}$ of $\mathrm{CsNO}_{3}$ in $1 \mathrm{~L}$ of Millipore water. $1 \mathrm{mg}$ of $c$-PBNPs/PVA 105 composite sheet was soaked in $10 \mathrm{~mL}$ of $1.0 \mathrm{ppm}$ Cs solution at $27^{\circ} \mathrm{C}$. Subsequently, the test solution was shacked for appropriate times (10, 30 and $100 \mathrm{~min}$ ) at 600 r.p.m using multi shaker (MS300, AS ONE Corporation., Japan). Then the $c$-PBNPs/PVA composite nanofiber sheets were removed and the solution was 
analyzed by ICP-MS. The adsorption rate $(A, \%)$ was calculated using the following equation:

$$
A=100-\frac{A_{b}}{A_{a}} \cdot 100
$$

where $A_{b}$ and $A_{\mathrm{a}}$ are the concentration of Cs before and after test, respectively.

\section{${ }_{10}$ Results and discussion}

\section{Optimization of electrospinning conditions}

In our very recent course of investigation, we have optimized the electrospinning conditions for the preparation of PVA nanofibers and also the effect of PVA solution properties on the spinnability 15 of PVA were studied. ${ }^{20}$ The same optimized conditions were adopted for the preparation of PVA nanofibers. In addition, to obtain a water-insoluble and highly hydrophilic PBNPs-based PVA composite nanofiber with a very fine morphology, other important parameters such as wt $\%$ of PVA, PBNPs and GA, and 20 cross-linking time were extensively optimized. Initially, the wt $\%$ of PVA was optimized and found that the morphology of the PVA fiber is highly depended on the concentration of PVA solution. A $12 \mathrm{wt} \%$ of PVA was found to be the optimum concentration since it produced continuous and homogeneous 25 fibers without beads. Whereas, when the concentration of PVA was $13 \mathrm{wt} \%$ or high, the beads and fibers coexisted in the SEM images (data not shown). Subsequently, the wt\% of PBNPs was optimized; as consequences, higher amount of $24 \mathrm{wt} \%$ PBNPs (based on PVA wt\%) could be successfully incorporated into the 30 PVA fibers (Fig. 1c). When the amount of PBNPs was $32 \mathrm{wt} \%$, the spinning was discontinuous and the morphology of the fibers were irregular and rough (Fig. 1d and Fig. 2e). This is probably due to the aggregation of PBNPs at the higher concentrations (Figs. 1 and 2). ${ }^{21}$ However, as expected, the average diameter of 35 the $c$-PBNPs/PVA composite nanofibers decreased with increasing the concentration of the PBNPs (Fig. 1e), and this value was found to be 260, 350, 320, 250 and $200 \mathrm{~nm}$ for the PBNPs concentrations of $0,8,16,24$ and $32 \mathrm{wt} \%$, respectively. It is clear that the electrical conductivity of the PBNPs-PVA 40 solution played a crucial role on the diameter of the PBNPs/PVA nanofibers (see Fig. S1b in supporting information). ${ }^{22}$ For more information about the properties of PBNPs/PVA solutions such as

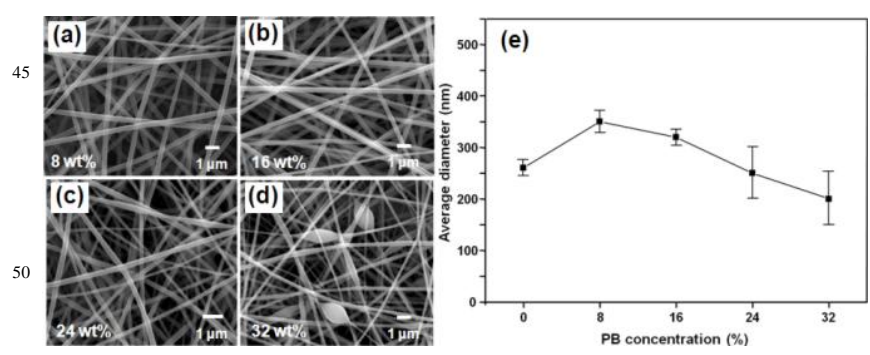

Fig. 1 SEM images of $c$-PBNPs/PVA composite fibers with PBNPs wt $\%$ of (a) $8 \%$, (b) $16 \%$, (c) $24 \%$ and (d) $32 \%$, and (e) ${ }_{55}$ fiber diameters of the corresponding $c$-PBNPs/PVA composite fibers.

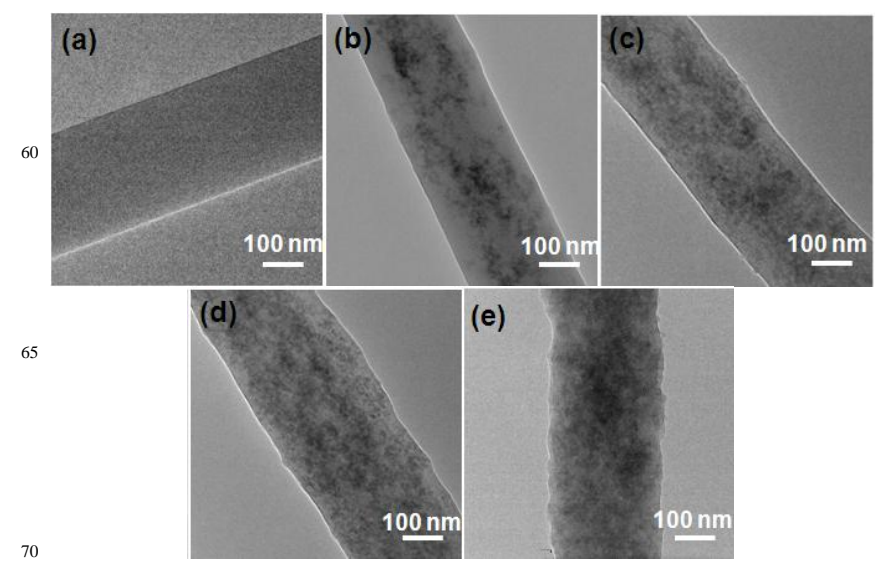

Fig. 2 TEM images of (a) pure PVA and $c$-PBNPs/PVA composite fibers with PBNPs wt $\%$ of (b) $8 \%$, (c) $16 \%$, (d) $24 \%$ and (e) $32 \%$.

75 viscosity, electrical conductivity and surface tension, please refer Fig. S1(a-c) in supporting information. Not surprisingly, as-spun pure PVA nanofibers are readily soluble in water at room temperature $\left(27^{\circ} \mathrm{C}\right)$. Therefore, to improve the water resistance property of composite nanofibers, we carried out a post cross80 linking process for the nanofiber composites using GA and $\mathrm{HCl}$ vapor as the cross-linker and catalyst, respectively. ${ }^{23}$ Prior to the cross-linking process, the wt $\%$ of GA $(2.5,7.5,12.5$ and 17.5 $\mathrm{wt} \%)$ and cross-linking time (10, 30 and $50 \mathrm{~s})$ were optimized. Interestingly, when the GA content was $12.5 \mathrm{wt} \%$ and the cross85 linking time was $30 \mathrm{~s}$, the fiber morphology and the average diameter of $c$-PBNPs/PVA were not changed much (see Fig. S2 in supporting information). Moreover, the FT-IR spectra and the water contact angle test showed that the $12.5 \mathrm{wt} \%$ of GA is good enough for the cross-linking of PVA (see Fig. S4 and Fig. S5 in 90 supporting information). Based on these results, the following solution properties and electrospinning conditions were shown to result in the formation of thinner and regular $c$-PBNPs/PVA composite fiber: $12 \mathrm{wt} \%$ of PVA, $24 \mathrm{wt} \%$ of PBNPs (based on PVA concentration), $12.5 \mathrm{wt} \%$ of GA and the cross-linking time 95 is $30 \mathrm{~s}$. The above resultant $c$-PBNPs/PVA composite fiber was characterized in detail by various spectroscopic and microscopic techniques. We also examined the electrospinning condition for the preparation of PBNPs/polyurethane and PBNPs/poly(vinylidene fluoride) composites, but homogeneous 100 solution was not obtained because of low compatibility of organic solvents ( $N, N$-dimethylformamide and methyl ethyl ketone) (data not shown).

\section{Characterization of $c$-PBNPs/PVA composite nanofiber}

105 The surface morphology of the $c$-PBNPs/PVA composite nanofiber was investigated by using SEM analysis. Fig. 3 shows the SEM images, digital photos (inset) and the fiber diameter distribution of pure PVA nanofibers and $c$-PBNPs/PVA composite nanofibers. It can be seen that the morphology of the 110 both pure PVA nanofibers (Fig. 3a) and $c$-PBNPs/PVA composite nanofibers (Fig. $3 \mathrm{~b}$ ) were smooth and continuous with diameters of 200-300 nm and lengths up to several millimeters. It is worth to mention that the fibrous shape and diameter of 

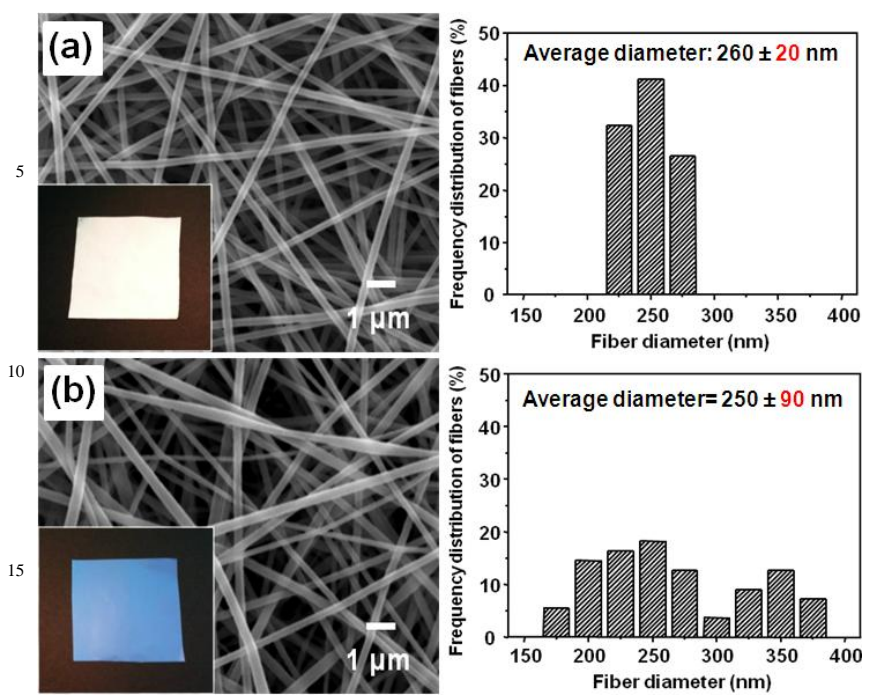

Fig. 3 SEM images (left) of (a) pure PVA nanofibers and (b) $c$ ${ }_{20}$ PBNPs/PVA composite nanofibers, and the fiber diameter distribution (right) of corresponding electrospun nanofibers.

$c$-PBNPs/PVA composite nanofibers maintained well even after the incorporation of PBNPs and cross-linking of GA. In addition, 25 no individual or aggregation of PBNPs was observed in the SEM image of $c$-PBNPs/PVA (Fig. 3b). The average fiber diameter of PVA nanofibers and $c$-PBNPs/PVA composite nanofibers are 260 $\pm 20 \mathrm{~nm}$ and $250 \pm 90 \mathrm{~nm}$, respectively. When compared to the results of pure PVA nanofibers, the fiber diameter slightly 30 decreased (from $260 \mathrm{~nm}$ to $250 \mathrm{~nm}$ ) and the distribution of fiber diameter too quite broaden (from $260 \pm 20 \mathrm{~nm}$ to $250 \pm 90 \mathrm{~nm}$ ). This is may be due to the higher electrical conductivity of the PBNPs/PVA/GA solution compared to PVA solution. Moreover, the inserted digital photos (Fig. 1a and 1b) show that the PVA 35 nanofibers are white color while $c$-PBNPs/PVA composite nanofibers are inherent blue color; confirms the successful incorporation PBNPs into the PVA matrix.

The well incorporation and homogeneous dispersion of PBNPs into the PVA matrix were investigated in detail by using TEM 40 analysis. Figure 4 shows the TEM images of pure PVA nanofibers (Fig. 4a) and $c$-PBNPs/PVA composite nanofibers (Fig. $4 \mathrm{~b}$ and $4 \mathrm{c}$ ). The results confirmed that the PBNPs are homogeneously distributed into the PVA nanofiber matrix (Fig. 4b). Most of the PBNPs are embedded within the PVA fiber, and 45 some of the PBNPs remain on the surface of the nanofibers (Fig. $4 c)$. The size of the incorporated PBNPs in $c$-PBNPs/PVA was
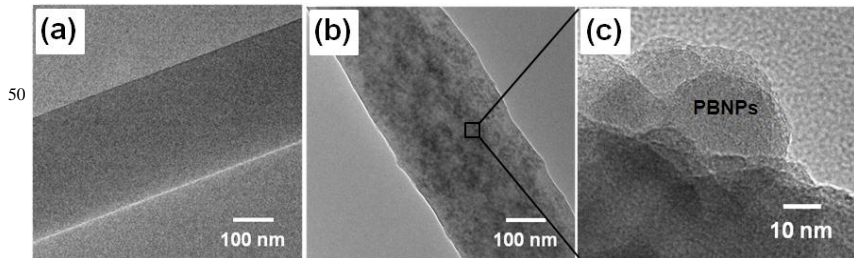

${ }_{55}$ Fig. 4 TEM images of (a) pure PVA nanofiber, (b) $c$ PBNPs/PVA composite nanofiber and (c) magnified TEM image of $c$-PBNPs/PVA composite nanofiber.

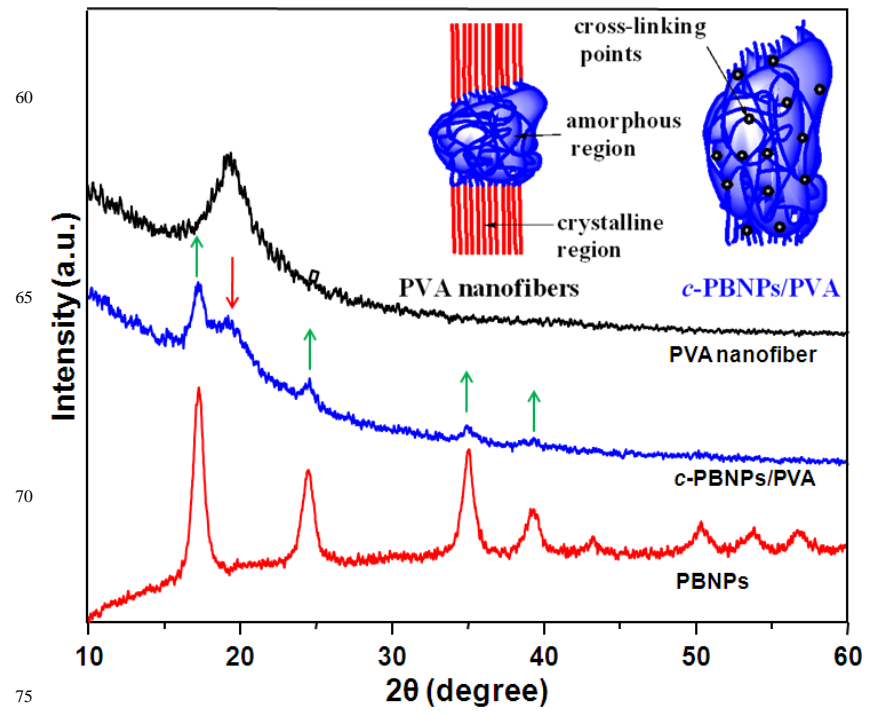

Fig. 5 XRD patterns of pure PVA nanofibers, PBNPs and $c$ PBNPs/PVA composite nanofibers (inset: schamatic illustarion of the crystaline and amorphose regions of PVA and $c$-PBNPs/PVA nanofibers).

80

found to be $\sim 15 \mathrm{~nm}$. Moreover, the PVA nanofibers exhibited smooth surface morphology (Fig. 4b), whereas the $c$-PBNPs/PVA composite nanofibers showed fairly rough surface morphology due to the incorporation of PBNPs.

85 Fig. 5 shows the XRD patterns of the pure PVA nanofibers, PBNPs and $c$-PBNPs/PVA composite nanofibers. The PBNPs displays several strong and broad peaks notably at $2 \theta=17.4^{\circ}$, $24.7^{\circ}, 35.3^{\circ}$ and $39.5^{\circ}$ correspond to typical crystal phases (200), (220), (400) and (420) of cubic face-centered PBNPs lattice 90 (JCPDS card no. 52-1907). ${ }^{23,24}$ For the electrospun PVA nanofibers, a significant crystalline peak at about $23.5^{\circ}(2 \theta)$ was observed, which is due to the strong intermolecular and intramolecular hydrogen bonding. ${ }^{25}$ In case of $c$-PBNPs/PVA composite nanofibers, the XRD spectrum is a combination of 95 pure PVA and PBNPs spectra. In detail, in the XRD spectrum of $c$-PBNPs/PVA composite nanofibers, except the diffraction peaks of PVA $\left[23.5^{\circ}(2 \theta)\right]$, all the other peaks corresponding to the PBNPs phase. The decrease in the peak intensity at $23.5^{\circ}$ for $c$ PBNPs/PVA composite nanofibers reveals a lower crystallinity of 100 the $c$-PBNPs/PVA composite when compared to PVA nanofibers. In fact, the suppression of PVA nanofibers crystallinity in the $c$ PBNPs/PVA composite fibers is mainly caused by destruction of the orientation order of the PVA chains and even by the formation of amorphous bound layers around the PBNPs. ${ }^{26}$ In the 105 present case, the homogeneously dispersed PBNPs act as steric hindrances within the composite fibers, and thus the crystallization is restricted by a decreasing amount of intact crystalline region. As a result, the usual planar zigazag conformation collapses and converts into an amorphous structure 110 on the interfacial region of PBNPs. Thus, the overall crystallinity of PVA is decreasing when the PBNPs are incorporated into the PVA matrix. This result is in good agreement with the TEM results (Fig. 5).

In order to confirm the cross-linking of PVA with GA, FT-IR 115 spectra were recorded for the PVA/PBNPs/GA 

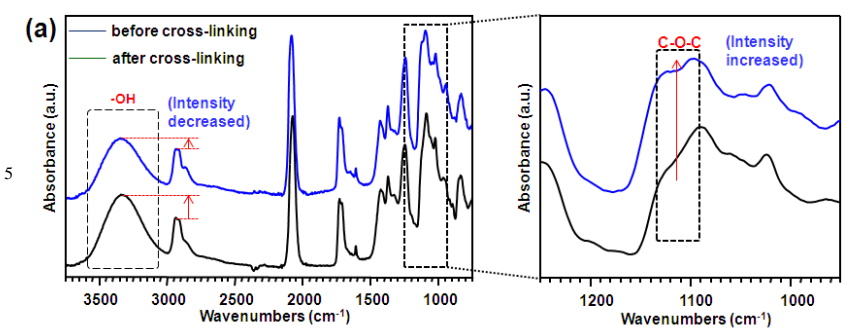

${ }_{10}$ (b)

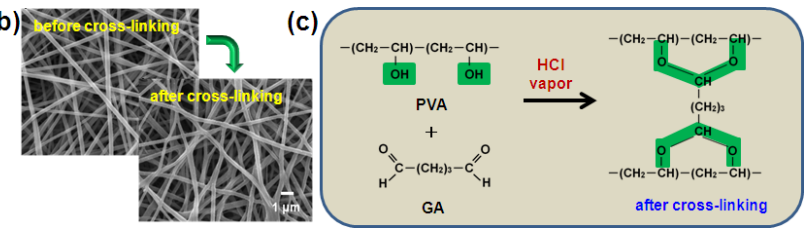

Fig. 6 (a) Full (left) and magnified (right) FT-IR spectra of $\mathrm{PBNPs} / \mathrm{PVA}$ composite nanofibers before (PVA/PBNPs/GA) and after corss-linking (c-PBNPs/PVA), (b) SEM images of a) $\mathrm{PVA} / \mathrm{PBNPs} / \mathrm{GA}$ and b) $c$-PBNPs/PVA composite nanofibers 20 (the scale bar is $1 \mu \mathrm{m}$ ), and (c) scheme showing the cross-linking of PVA with $\mathrm{GA}$ under $\mathrm{HCl}$ vapor.

(before cross-linking) and $c$-PBNPs/PVA (after cross-linking) composites nanofibers (Fig. 6). As expected, both the FT-IR 25 spectra exhibited characteristic peaks of PVA and PBNPs. In Fig. $6 \mathrm{a}$, the characteristic absorption bands of PVA were absorbed at $3278 \mathrm{~cm}^{-1}$ (stretching of $-\mathrm{OH}$ group), $2935 \mathrm{~cm}^{-1}$ ( $v_{\text {as }}$ of $-\mathrm{CH}_{2}$ ), $2906 \mathrm{~cm}^{-1}$ ( $v_{\mathrm{s}}$ of $-\mathrm{CH}_{2}$ ), $1417 \mathrm{~cm}^{-1}$ (bending of $-\mathrm{OH}$ and wagging of $-\mathrm{CH}_{2}$ ), $1143 \mathrm{~cm}^{-1}$ (stretching of $-\mathrm{C}-\mathrm{O}-\mathrm{C}-$ from crystalline 30 sequence of PVA), $1088 \mathrm{~cm}^{-1}$ (stretching of $\mathrm{CO}$ and bending of $\mathrm{OH}$ from amorphous sequence of PVA), $919 \mathrm{~cm}^{-1}$ (bending of $\mathrm{CH}_{2}$ ) and $838 \mathrm{~cm}^{-1}$ (rocking of $-\mathrm{CH}$ ). ${ }^{27}$ Similarly, the FT-IR spectrum of the samples exhibited a peak at $2110 \mathrm{~cm}^{-1}$ attributed to the $-\mathrm{C} \equiv \mathrm{N}$ stretching in the formed [FeII-CN-FeIII] structure, ${ }_{35}$ which indicates the presence of PBNPs. ${ }^{28}$ Apart from the characteristic absorption band, there are two significant changes in the FT-IR peaks (-OH and $-\mathrm{C}-\mathrm{O}-\mathrm{C}-$ ) obviously confirm the cross-linking of PVA with GA. ${ }^{29}$ In the FI-IR spectrum of $c$ PBNPs/PVA composites nanofibers, the intensity of the 40 adsorption peak at $3300 \mathrm{~cm}^{-1}$ (-OH stretching) decreased and simultaneously the adsorption peak at $1150-1085 \mathrm{~cm}^{-1}(-\mathrm{C}-\mathrm{O}-\mathrm{C}-$ stretching) increased when compared with that of the uncrosslinked nanocomposite nanofibers (PVA/PBNPs/GA). These results confirm that the $-\mathrm{OH}$ groups in the PVA and the $-\mathrm{CHO}$ ${ }_{45}$ groups in GA typically reacted to form an acetal groups or ether linkages during the cross-linking process (Fig. 6c). ${ }^{30}$

Several existing PBNPs-based nanocomposites highly suffer from the leaching of PBNPs after the adsorption of Cs from the wastewater and sometimes the nanocomposites are partially 50 soluble in water, which leads to low environmental impact and therefore hinders its direct industrial applications. Hence, the water resistance property and the stability of $c$-PBNPs/PVA composite nanofibers were investigated in detail. In a typical experiment, $1 \mathrm{~g}$ of $c$-PBNPs/PVA composite nanofibers was 55 dipped into a $50 \mathrm{~mL}$ of Milli Q water and shaked for $100 \mathrm{~min}$ using a multi shaker with $600 \mathrm{rpm}$ at $27^{\circ} \mathrm{C}$. Finally, the mixture was filtered and the leaching of Fe was analyzed by ICP-MS. Fig. 7 show digital photos of $c$-PBNPs/PVA composites nanofiber
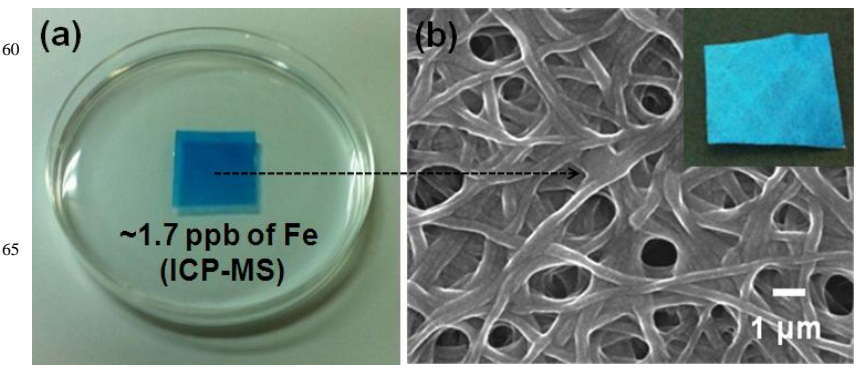

Fig. 7 Digital photos of (a) $c$-PBNPs/PVA composite nanofibers 70 soaked in distilled water and (b) SEM image (inset: digital photo) of cross-linked $c$-PBNPs/PVA composite nanofibers after soaking in distilled water for $1 \mathrm{~h}$ (dried).

soaked in distilled water for $1 \mathrm{~h}$ at $27^{\circ} \mathrm{C}$. As seen from Fig. 7, no 75 significant leaching of PBNPs from $c$-PBNPs/PVA composite nanofibers is observed. In addition, after soaking, the soaked water and the composites were further analyzed by ICP-MS analysis. The results confirmed that the $c$-PBNPs/PVA composite nanofibers are highly stable since it showed very less amount of $80(\sim 1.7 \mathrm{ppb})$ leaching of PBNPs and the unaffected morphology of $c$-PBNPs/PVA composite nanofibers (See Table S1 in Supporting information) ${ }^{31}$ This is may be due to the effective cross-linking process of PVA with GA.

Since the hydrophilic nature of the $c$-PBNPs/PVA composite 85 nanofibers is one of the highly significant properties for the rapid adsorption of the Cs, the wettability of the pure PVA and $c$ PBNPs/PVA-composite nanofibers were investigated by the measurements of water contact angles. In Fig. 8, the photographs show the water contact angles of Teflon sheet (for reference), 90 pure PVA nanofibers and $c$-PBNPs/PVA composite nanofibers. Although the surface of the $c$-PBNPs/PVA composite nanofibers are rough, it showed lower water contact angle of $29^{\circ}$ than that of pure PVA nanofibers $\left(91^{\circ}\right)$, which obviously indicates that the cross-linked $c$-PBNPs/PVA composite have high hydrophilic 95 nature. This is due to the presence of unreacted (uncross-liked) $\mathrm{OH}$ and $-\mathrm{CHO}$ groups in the $c$-PBNPs/PVA composite nanofibers. ${ }^{32}$ In addition, the high hydrophilic nature of

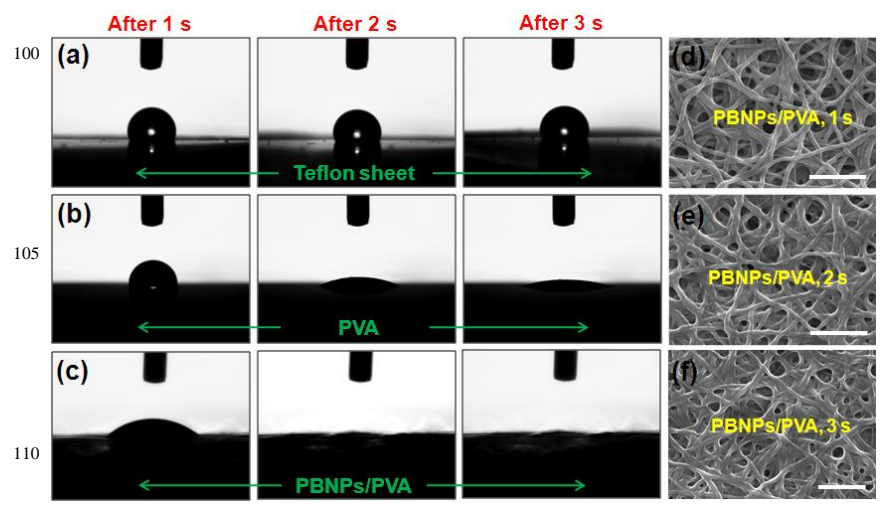

Fig. 8 Pictures showing the water contact angles of (a) Teflon sheet (for reference), (b) pure PVA nanofibers with a smooth surface, (c) $c$-PBNPs/PVA composite nanofibers with a rough 115 surface, and SEM images of $c$-PBNPs/PVA after the water contact angle test; after (d) $1 \mathrm{~s}$, (e) $2 \mathrm{~s}$ and (f) $3 \mathrm{~s}$. 
the $c$-PBNPs/PVA composite is may also be due to the amorphous nature of the $c$-PBNPs/PVA composite when compared to the PVA nanofibers. The result agrees well with the SEM and XRD results (Fig. 3 and Fig. 4).

\section{Evaluation of Cs adsorption performance}

After the extensive characterization, the $c$-PBNPs/PVA composite nanofiber mat was used for the removal of Cs from the wastewater. ICP-MS analysis was used to study the adsorption 10 activity of the $c$-PBNPs/PVA. Initially, the Cs adsorption behavior of the pure PVA nanofiber was investigated and it was found to be very low even after the soaking time of $100 \mathrm{~min}$. Fig. 6 shows the $\mathrm{Cs}$ adsorption rate of $c$-PBNPs/PVA composite nanofibers. The adsorption rate of $c$-PBNPs/PVA increased with 15 increasing soaking time, and this value was found to be $86 \%$, $90 \%$, and $96 \%$ for the soaking time of $10 \mathrm{~min}, 30 \mathrm{~min}$, and 100 min, respectively. The merit of the present $c$-PBNPs/PVA was
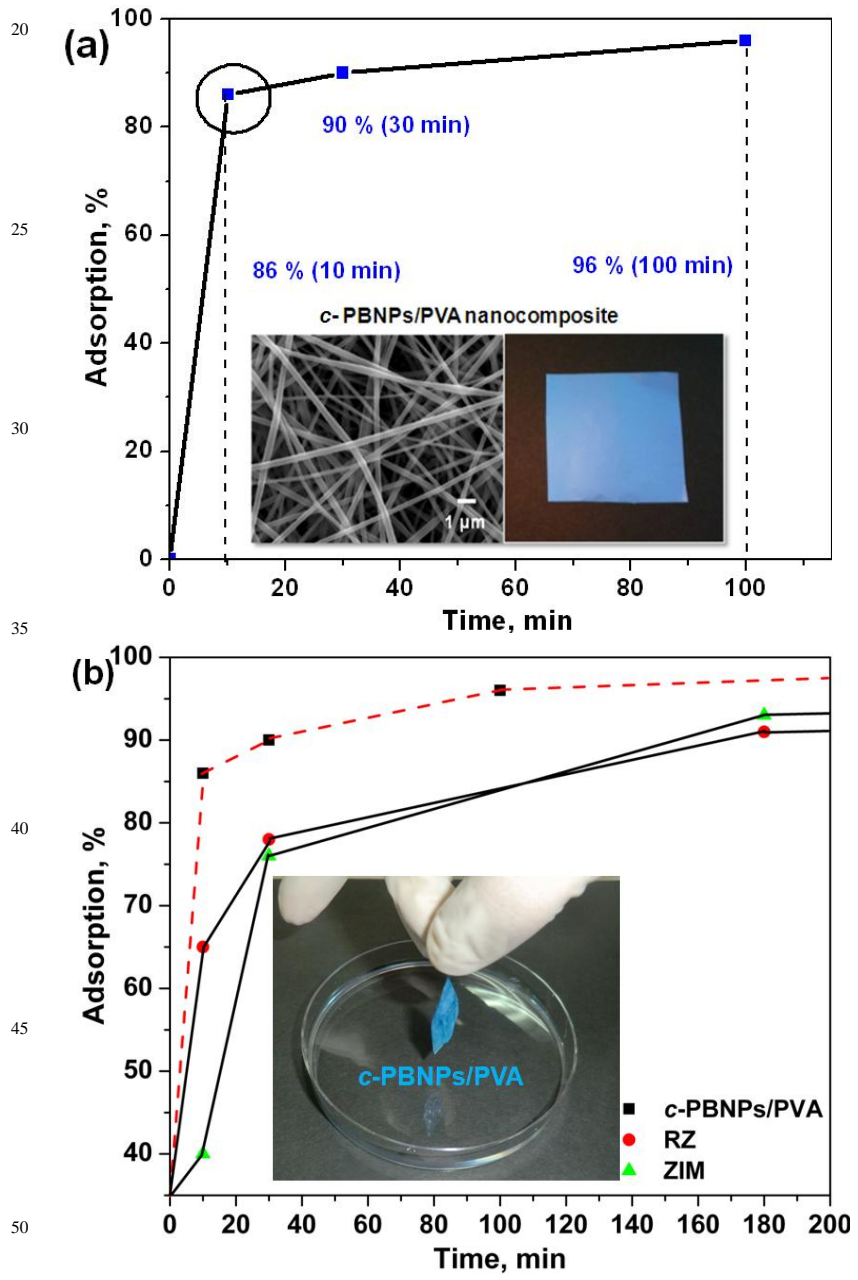

Fig. 9 (a) Adsorption of $\mathrm{Cs}$ onto $c$-PBNPs/PVA composite nanofiber at different time intervals and (b) comparison of $\mathrm{Cs}$ adsorption activity of present composite nanofiber (c55 PBNPs/PVA) with previously reported nanocmposites (RZ [10] and ZIM [40]) (inset showing the separation of $c$-PBNPs/PVA after treatment). realized from the very high adsorption rate of $86 \%$ after only 10 min. To the best of our knowledge, this is the highest activity ${ }_{60}(86 \%$ after $10 \mathrm{~min})$ reported for the adsorption of $\mathrm{Cs}$ from the radioactive wastewater till to date. Moreover, the results are well comparable to those previous reported. In most of the previous studies, the hybrid materials have showed the maximum adsorption rate of about 30-65\% after the soaking time of 10 ${ }_{65}$ min. $^{33-39}$ For instance, the magnetic hexacyanoferrate (II) polymeric nanocomposite (RZ) showed the adsorption rate of $\sim 65 \%$ after $10 \mathrm{~min}$ and the maximum adsorption rate of $\sim 95 \%$ only after $150 \mathrm{~h},{ }^{10}$ whereas the present $c$-PBNPs/PVA composite nanofibers showed a very higher Cs adsorption rate of $\sim 96 \%$ after 70 only a very less soaking time of $100 \mathrm{~min}(1.7 \mathrm{~h})$. Similarly, the present $c$-PBNPs/PVA composite nanofibers confirmed a better adsorption rate of $\sim 96 \%$ (100 $\mathrm{min})$ when compared to the zirconium(IV) iodomolybdate exchanger (ZIM) (Fig. 8). ${ }^{40}$ The results confirm the higher activity of the present $c$-PBNPs/PVA 75 composite nanofibers towards the adsorption of radioactive $\mathrm{Cs}$ from the wastewater. From the value of Cs adsorption rate, it was calculated that $1 \mathrm{~g}$ of $c$-PBNPs/PVA composite is enough to remove $\sim 8 \mathrm{mg}$ of $\mathrm{Cs}$ from the wastewater. Although the value is not higher when compared to the previous reports [9, 10, 40-44],

80 the proposed composite material has shown a faster Cs adsorption rate in addition to the other advantages such as easy-handling and separation. There are three possible reasons for the higher activity of this newly developed $c$-PBNPs/PVA composite nanofibers: (1) higher surface area and the three dimensional structure of the $c$ ${ }_{85}$ PBNPs/PVA composite nanofibers web, (2) higher hydrophilic nature of the composite and (3) water-insoluble property of the $c$ PBNPs/PVA composite nanofibers. Apart from these most possible reasons, the higher dispersion of PBNPs into the PVA matrix might also be assisted for the higher adsorption rate of $\mathrm{Cs}$ 90 from the wastewater.

\section{Separation of $c$-PBNPs/PVA after Cs adsorption test}

The separation of Cs adsorbents after treatment is very important since the radioactive $\mathrm{Cs}$ is highly harmful. ${ }^{9,13,14}$ At present, the 95 challenging task is the development of easily separable Cs adsorbents. $^{45}$ There are several reports describe various separation techniques for the removal Cs adsorbents after treatment. However, since the PBNPs-based nanocomposites are very small in size $(10-100 \mathrm{~nm})$, the separation process is more 100 complicated and expensive. For instance, Chakrit et al., ${ }^{9}$ reported that the PBNPs-coated magnetic NPs for the removal of Cs from wastewater. However, they used a neodymium magnet (strength of $0.25 \mathrm{~T}$ ) for the separation of PBNPs-coated MNP nano-sorbent from the wastewater after treatment. Similarly, magnetic $105 \mathrm{PBNPs} /$ graphene oxide nanocomposites (PBNPs/GO) for the removal of radioactive $\mathrm{Cs}$ were reported by Hongjun and coworkers. $^{14}$ In spite of their higher adsorption activity, the separation of PBNPs/GO is very difficult and expensive. Interestingly, in the present study, the separation of Cs adsorbent 110 (c-PBNPs/PVA composite nanofiber) is very simple and efficient. After the Cs adsorption test, the $c$-PBNPs/PVA composite nanofiber sheets can be easily taken off from the wastewater by well-gloved hands (inset in Fig. 9b). The simple and effective 
separation of $c$-PBNPs/PVA composite nanofiber is also one of the hall marks of this work. Since the handling and separation are very easy, the present $c$-PBNPs/PVA composite may be good to use for the removal of Cs particularly from the seawater and 5 groundwater.

\section{Conclusions}

In summary, we have successfully prepared a new PBNPs-based PVA composite nanofibers ( $c$-PBNPs/PVA) via electrospinning. ${ }_{10} \mathrm{SEM}$ images revealed smooth and continuous nanofiber morphology of the $c$-PBNPs/PVA composite with diameters of 200-300 nm and lengths up to several millimeters. TEM images confirmed homogeneous dispersion and well incorporation of PBNPs into the PVA matrix. The amorphous nature of the $c$ 15 PBNPs/PVA composite nanofibers was confirmed by the XRD analysis. FT-IR spectra showed successful cross-linking of PVA with GA. It was found that the prepared nanocomposite fiber is highly hydrophilic and water-insoluble. The excellent activity of the $c$-PBNPs/PVA composite nanofibers can be realized from the 20 higher Cs adsorption rate of $96 \%$ after only $100 \mathrm{~min}$. Moreover, the mass production of the $c$-PBNPs/PVA is simple and costeffective. After Cs adsorption test, the $c$-PBNPs/PVA can be easily separated from the wastewater. Overall, the simple preparation, easy separation and faster Cs adsorption activity 25 make $c$-PBNPs/PVA as an alternate choice to the existing PBNPs-composite materials.

\section{Notes and references}

${ }^{a}$ Nano Fusion Technology Research Lab, Division of Frontier 30 Fibers, Institute for Fiber Engineering (IFES), Interdisciplinary Cluster for Cutting Edge Research (ICCER), National University Corporation, Shinshu University, Ueda, Nagano 386 8567, Japan. Fax: +81 26821 5482,Tel: +8126821 5139, E-mail: kim@shinshu-u.ac.jp, kimicksoo.gr@gmail.com

$35{ }^{b}$ College of Textile Clothing Engineering, Soochow University, Suzhou, 215021, China

${ }^{c}$ Department of Clothing \& Textiles, PaiChai University, Daejeon 302735, South Korea

${ }^{d}$ Department of Clothing and Textiles, Chungnam National University, 40 Daejeon 305-764, South Korea

$\dagger$ Electronic Supplementary Information (ESI) available: SEM images, FT-IR spectra and ICP-MS results. See DOI: 10.1039/b000000x/

45

1 M. Yu, O. Toshimasa, N. Masato, J. Geophys. Res., 2011, 38, 1-7.

2 K. Hiroaki, O. Yuichi, T. Mengistu, J. Environ. Radioactiv., 2012, 111, 59-64.

3 K. Hideyuki, K. Takuya, F. Akiko, I. Teiji, I. Yoichi, N. Tomoharu, S

$50 \quad$ Shigeki, A. Toshiyuki, J. Nucl. Sci. Technol., 2011, 48, 1349-1356.

4 C. Masamichi, N. Hiromasa, N. Haruyasu, T. Hiroaki, K. Genki, Y. Hiromi, J. Nucl. Sci. Technol., 2011, 48, 1129-1134.

5 S. Thanapon, S. Vichaya, J. W. Robert, M. G. Rafal, E. F. Glen, A. R. Shane, T. Charles, Y. Wassana, J. Hazard. Mater., 2010, 182, 22555231.

6 L. T. Nagy, H. Ming, I. Masataka, N. Masanobu, Y. Yusuke, J. Mater. Chem., 2012, 22, 18261-18267.

7 A. de la Escosura, V. Martijn, D. S. Friso, C. A. Marta, K. Andrei, R.
Theo, J. M. N. Roeland, J. L. M. C. Jeroen, Chem. Commun., 2008 , $60 \quad 1542-1544$.

8 T. Subbiah, G. S. Bhat, R. W. Tock, S. Parameswaran, S. S. Ramkumar, J. Appl. Poly. Sci., 2005, 96, 557-569.

9 T. Chakrit, O. Pakorn, T. Pramuan, S. Paiboon, J. Nanopart. Res., 2013, 15, 1689(1-10).

6510 R. S. Reda, J. Colloid Interface Sci., 2012, 388, 21-30.

11 M. Gopiraman, R. Karvembu, I. S. Kim, ACS Catal., 2014, 4, 2118-2129.

12 M. Gopiraman, S. Ganesh Babu, Z. Khatri, K. Wei, Y. A. Kim, M. Endo, R. Karvembu, I. S. Kim, J. Phys. Chem. C, 2013, 117, 23582 23596.

13 Y. Hongjun, S. Lei, Z. Jiali, L. Haiyan, Z. Yan, Y. Hongwen, $J$. Mater. Chem. A, 2014, 2, 326-332.

14 M. Gopiraman, S. Ganesh Babu, Z. Khatri, W. Kai, Y. A. Kim, M. Endo, R. Karvembu, I. S. Kim. Carbon, 2013, 62, 135-148.

7515 O. Ohsawa, K. H. Lee, B. S. Kim, S. Lee, I. S. Kim, Polymer, 2010 51, 2007-2012.

16 S. Park, H. R. Kim, H. Bang, K. Fujimori, B. S. Kim, S. H. Kim, I. S. Kim, J. Appl. Poly. Sci., 2012, 125, 2929-2935.

17 H. Dian, H. Bo, Y. Qiao-Feng, W. Kan, Y. Shu-Hong, ACS Nano, $80 \quad 2009,3,3993-4002$.

18 B. Jie, L. Yaoxian, Y. Songtao, D. Jianshi, W. Shugang, Z. Jifu, W. Yongzhi, Y. Qingbiao, C. Xuesi, J. A. Xiabin, Solid State Chem., 2007, 141, 292-295.

19 Z. H. Mbhele, M. G. Salemane, C. G. C. E. van Sittert,J. M. 85 Nedeljkovic, V. Djokovic, A. S. Luyt, Chem. Mater., 2003, 15, 5019 5024.

20 J. C. Park, T. Ito, K. O. Kim, K. W. Kim, B. S. Kim, M. S. Khil, H. Y. Kim, I. S. Kim, Polymer Journal, 2010, 42, 273-276.

21 K. Hyeyoung, J. Jyongsik, Chem. Commun. 2006, 3010-3012.

9022 Z. Chuan-Ling, L. Kong-Peng, C. Huai-Ping, Y. Shu-Hong, Small, 2012, 8, 648-653.

23 K. O. Kim, Y. Akada, K. Wei, B. S. Kim, I. S. Kim, J. Biomater. Nanobiotechn., 2011, 2, 353-360.

24 T. Chakrit, O. Pakorn, T. Pramuan, S. Paiboon, J. Nanopart. Res., $95 \quad 2013,15,1689(1-10)$.

25 J. H. Park, M. R. Karim, I. K. Kim, I. W. Cheong, J. W. Kim, D. G. Bae, J. W. Cho, J. H. Yeum, Colloid Polym. Sci., 2010, 288, 115-121.

26 S. K. Chang, H. B. Doo, D. G. Kyung, H. L. Ki, C. U. In, H. P. Young, Polymer, 2005, 46, 5094-5102.

10027 H. S. Mansur, C. M. Sadahira, A. N. Souza, A. A. Mansur, Mater. Sci. Eng. C, 2008, 28, 539-548.

28 J. K. Pawel, A. M. Marcin, D. Andrzej, S. Jerzy, Anal. Chem., 1996, 68, 2442-2446.

29 S. M. Herman, L. O. Rodrigo, A. P. M. Alexandra, Polymer, 2004, 45, $105 \quad 7193-7202$.

30 C. Tang, C. D. Saquing, J. R. Harding, S. A. Khan, Macromolecules, 2010, 43, 630-637.

31 M. Gopiraman, H. Bang, S. Ganesh Babu, K. Wei, R. Karvembu, I. S. Kim, Catal. Sci. Technol., 2014, 4, 2099-2106.

11032 K. Fujimori, M. Gopiraman, H. K. Kim, B. S. Kim, I. S. Kim, J. Nanosci. Nanotechno., 2013, 13, 1759-1764.

33 Z. Q. Zhang, S. W. Gong, Z. Yu, Y. Ting, C. Y. Wang, N. Gu, J. Mater. Chem., 2010, 20, 5110-5116.

34 X. Liu, G. R. Chen, D. J. Lee, T. Kawamoto, H. Tanaka, M. L. Chen, 115 Y. K. Luo, Bioresour. Technol., 2014, 160, 142-149.

35 P. Durga, T. Hisashi, H. Yukiya, M. Kimitaka, F. Shigeharu, U. Kuniyoshi, K. Ryuichi, H. Yukie, O. Masatoshi, K. Tohru, Environ. Sci. Technol., 2013, 47, 3800-3806.

36 M. Ishizaki, S. Akiba, A. Ohtani, Y. Hoshi, K. Ono, M. Matsuba, T. 120 Togashi, K. Kananizuka, M. Sakamoto, A. Takahashi, T. Kawamoto, H. Tanaka, M. Watanabe, M. Arisaka, T. Nankawa, M. Kurihara, Dalton Trans., 2013, 42, 16049-16055.

37 H. Baiyang, F. Bunshi, Y. Hongwen, A. Yoshiteru, J. Hazard. Mater., 2012, 217-218, 85-91.

12538 H. H. Kouji, F. Yukiko, A. Ayumu, T. Ayako, A. Fumikazu, K. Akio, Environ. Sci. Technol., 2013, 47, 2520-2526.

39 I. Damir, G. Andreja, C. Miran, P. Boris, Electroanalysis, 2010, 22, 2202-2210. 
40 R. R. Sheha, S. H. El-Khouly, Chem. Eng. J., 2013, 91, 942-954.

41 A. Nilchi, R. Saberi, M. Moradi, H. Azizpour, R. Zarghami, Chem. Eng. J., 2011, 172, 572-580.

42 E.H. Borai, R. Harjula, L. Malinen, A. Paajanen, J. Hazard. Mater., 2009, 172, 416-422.

43 A. Abusafa, H. Yucel, Sep. Purif. Technol., 2002, 28, 103-116.

44 B. Yıldız, H.N. Erten, M. Kis, J. Radioanal. Nucl. Chem., 2011, 288, 475-483.

45 R. R. Sheha, S. H. El-Khouly, Adsorpt. Sci. Technol., 2011, 29, 139-

10 155 . 